\title{
Mixable shuffles, quasi-shuffles and Hopf algebras
}

\author{
Kurusch Ebrahimi-Fard · Li Guo
}

Received: 20 June 2005 / Accepted: 27 December 2005

(C) Springer Science + Business Media, LLC 2006

\begin{abstract}
The quasi-shuffle product and mixable shuffle product are both generalizations of the shuffle product and have both been studied quite extensively recently. We relate these two generalizations and realize quasi-shuffle product algebras as subalgebras of mixable shuffle product algebras. As an application, we obtain Hopf algebra structures in free Rota-Baxter algebras.
\end{abstract}

\section{Introduction}

This paper studies the relationship between the mixable shuffle product and the quasishuffle product, both generalizations of the shuffle product.

Mixable shuffles arise from the study of Rota-Baxter algebras. Let $\mathbf{k}$ be a commutative ring and let $\lambda \in \mathbf{k}$ be fixed. A Rota-Baxter $\mathbf{k}$-algebra of weight $\lambda$ (previously called a Baxter algebra) is a pair $(R, P)$ in which $R$ is a $\mathbf{k}$-algebra and $P: R \rightarrow R$ is a k-linear map, such that

$$
P(x) P(y)=P(x P(y))+P(P(x) y)+\lambda P(x y), \forall x, y \in R .
$$

The concept of Rota-Baxter algebra was introduced by the mathematician Glen Baxter [3] in 1960 to study the theory of fluctuations in probability. It was motivated by the work of Spitzer on random walks [41]. Rota greatly contributed to the study of the Rota-Baxter algebra by his pioneer work in the late 1960s and early 1970s [36, 37, 38] and by his survey articles in late 1990s [39, 40]. Unaware of these works, in the early

Kurusch Ebrahimi-Fard $(\square)$

Universität Bonn - Physikalisches Institut, Nussallee 12, D-53115 Bonn, Germany

email: kurusch@ihes.fr

Li Guo

Department of Mathematics and Computer Science, Rutgers University, Newark, NJ 07102, USA

email: liguo@newark.rutgers.edu 
1980s the school around Faddeev, especially Semenov-Tian-Shansky [42], developed a whole theory for the Lie algebraic version of equation (1), which is nowadays wellknow in the realm of the theory of integrable systems under the name of the (modified) classical Yang-Baxter equation. ${ }^{1}$ In recent years, Rota-Baxter algebras have found applications in quantum field theory $[8,9,15,16,17]$, dendriform algebras $[1,10,13$, 31], number theory [22], Hopf algebras [2] and combinatorics [21].

Key to many of these applications is the realization of the free objects in which the product is defined by mixable shuffles [23, 24] as a generalization of the shuffle product. The shuffle product is a natural generalization of the integration by parts formula and its construction can be traced back to Chen's path integrals [7] in 1950s. It has been defined and studied in many areas of mathematics, such as Lie and Hopf algebras, algebraic $K$-theory, algebraic topology and combinatorics. Its applications can also be found in chemistry and biology. It naturally carries the notion of a RotaBaxter operator of weight zero.

Another paper [26] on a generalization of the shuffle product, called the quasishuffle product, was published by Hoffman ${ }^{2}$ [26] in the same year as the papers [23, 24] on mixable shuffle products. Hoffman's quasi-shuffle product plays a prominent role in recent studies of harmonic functions, quasi-symmetric functions, multiple zeta values $[25,27,28,4]$ (where in special cases it is also called stuffle product or harmonic product) and $q$-multiple zeta values [5].

Despite the extensive works on the two generalizations of shuffle products, it appears that they were carried out without being aware of each other. In particular, the relation of quasi-shuffles with Rota-Baxter algebras seems unnoticed. For example, in the numerous applications of quasi-shuffles to multiple zeta values in the current literature, no connections with Rota-Baxter algebras and mixable shuffles have been mentioned. In fact, concepts and results on Rota-Baxter algebras were rediscovered in the study of multiple zeta values. For instance, the construction of the stuffle product in [5] follows easily from the construction of free Rota-Baxter algebras in [6], while the generalized shuffle product in [19] is the same as the mixable shuffle product in [23, 24].

The situation is similar in the theory of dendriform algebras. Even though both quasi-shuffles and Rota-Baxter algebras have been used to give examples of dendriform algebras [1, 10, 12, 33], no connection of the two has been made. Also, in the work of Kreimer, and Connes and Kreimer [29, 30, 8, 9] on renormalization theory in perturbative quantum field theory, both the shuffle and its generalization in terms of the quasi-shuffle, and Rota-Baxter algebras appeared, in different contexts.

It was noted in [11] that the two constructions should be related. Our first goal of this paper is to make this connection precise. We show that the recursive formula for the quasi-shuffle product has its explicit form in terms of the mixable shuffle product. Both can be derived from the Baxter relation (1) that defines a Rota-Baxter algebra of weight 1 . We further show that the quasi-shuffle algebra on a locally finite set, to be recalled below, is a subalgebra of a mixable shuffle algebra on the corresponding locally finite algebra. With this connection, the concept of quasi-shuffle algebras can be defined for a larger class of algebras.

\footnotetext{
${ }^{1}$ The latter Baxter is the Australian physicist Rodney Baxter.

${ }^{2}$ Hoffman mentioned in [26] that there was also a generalization in the thesis of F. Fares [18]. 
This connection allows us to use the Hopf algebra structure on quasi-shuffle algebras to obtain Hopf algebra structures on free Rota-Baxter algebras, generalizing a previous work [2] on this topic. In the other direction, considering the critical role played by the quasi-shuffle (stuffle) product in recent work on multiple zeta values and quasisymmetric functions, this connection should allow us to use the theory of Rota-Baxter algebras in the studies of these exciting areas [14].

The paper is organized as follows. In the next section, we recall the concepts of shuffles, quasi-shuffles and mixable shuffles, and describe their relations (Theorem 2.5). In Section 3, we use these connections to obtain Hopf algebra structures on free Rota-Baxter algebras (Theorem 3.3).

\section{Shuffles, quasi-shuffles, and mixable shuffles}

For the convenience of the reader and for the ease of later references, we recall the definition of each product before giving the relation among them.

\subsection{Shuffle product}

The shuffle product can be defined in two ways, one recursively, one explicitly. We will see that Hoffman's quasi-shuffle product is a generalization of the recursive definition and the mixable shuffle product is a generalization of the explicit definition.

Let $\mathbf{k}$ be a commutative ring with identity $\mathbf{1}_{\mathbf{k}}$. Let $V$ be a $\mathbf{k}$-module. Consider the k-module

$$
T(V)=\bigoplus_{n \geq 0} V^{\otimes n}
$$

Here the tensor products are taken over $\mathbf{k}$ and we take $V^{\otimes 0}=\mathbf{k}$.

Usually the shuffle product on $T(V)$ starts with the shuffles of permutations [35, 43]. For $m, n \in \mathbb{N}_{+}$, define the set of $(m, n)$-shuffles by

$$
S(m, n)=\left\{\begin{array}{l|l}
\sigma \in S_{m+n} & \begin{array}{l}
\sigma^{-1}(1)<\sigma^{-1}(2)<\ldots<\sigma^{-1}(m), \\
\sigma^{-1}(m+1)<\sigma^{-1}(m+2)<\ldots<\sigma^{-1}(m+n)
\end{array}
\end{array}\right\} .
$$

Here $S_{m+n}$ is the symmetric group on $m+n$ letters.

For $a=a_{1} \otimes \ldots \otimes a_{m} \in V^{\otimes m}, b=b_{1} \otimes \ldots \otimes b_{n} \in V^{\otimes n}$ and $\sigma \in S(m, n)$, the element

$$
\sigma(a \otimes b)=u_{\sigma(1)} \otimes u_{\sigma(2)} \otimes \ldots \otimes u_{\sigma(m+n)} \in V^{\otimes(m+n)}
$$

where

$$
u_{k}= \begin{cases}a_{k}, & 1 \leq k \leq m \\ b_{k-m}, & m+1 \leq k \leq m+n\end{cases}
$$


is called a shuffle of $a$ and $b$. The sum

$$
a \amalg b:=\sum_{\sigma \in S(m, n)} \sigma(a \otimes b)
$$

is called the shuffle product of $a$ and $b$. Also, by convention, $a \amalg b$ is the scalar product if either $m=0$ or $n=0$. The operation $\amalg$ extends to a commutative and associative binary operation on $T(V)$, making $T(V)$ into a commutative algebra with identity, called the shuffle product algebra generated by $V$, which we denote by the pair $(T(V), U)$. It is well-know [43] that, when $V$ is a vector space over a field $\mathbf{k}$, then $(T(V), \amalg)$ is endowed with a coproduct (deconcatenation), making it into a Hopf algebra.

The shuffle product on $T(V)$ can also be recursively defined as follows. As above we choose two elements $a_{1} \otimes \cdots \otimes a_{m} \in V^{\otimes m}$ and $b_{1} \otimes \cdots \otimes b_{n} \in V^{\otimes n}$, and define

$$
\begin{aligned}
& a_{0} \amalg\left(b_{1} \otimes b_{2} \otimes \ldots \otimes b_{n}\right)=a_{0} b_{1} \otimes b_{2} \otimes \ldots \otimes b_{n}, \\
& \left(a_{1} \otimes a_{2} \otimes \ldots \otimes a_{m}\right) \amalg b_{0}=b_{0} a_{1} \otimes a_{2} \otimes \ldots \otimes a_{m}, a_{0}, b_{0} \in V^{\otimes 0}=\mathbf{k},
\end{aligned}
$$

and

$$
\begin{aligned}
\left(a_{1} \otimes \ldots \otimes a_{m}\right) \amalg\left(b_{1} \otimes \ldots \otimes b_{n}\right) & \\
= & a_{1} \otimes\left(\left(a_{2} \otimes \ldots \otimes a_{m}\right) \amalg\left(b_{1} \otimes \ldots \otimes b_{n}\right)\right) \\
& \quad+b_{1} \otimes\left(\left(a_{1} \otimes \ldots \otimes a_{m}\right) \amalg\left(b_{2} \otimes \ldots \otimes b_{n}\right)\right), a_{i}, b_{j} \in V .
\end{aligned}
$$

Lemma 2.1. For every element $v \in V$, the k-linear map $P_{(v)}:(T(V), \amalg) \rightarrow$ $(T(V), \amalg), P_{(v)}(a):=v \otimes$ a is a Rota-Baxter operator of weight zero.

Proof: Let $a:=a_{1} \otimes \cdots \otimes a_{m} \in V^{\otimes m}$ and $b:=b_{1} \otimes \cdots \otimes b_{n} \in V^{\otimes n}$. It is evident from the recursive definition of the shuffle product in (3) that $P_{(v)}(a) \amalg P_{(v)}(b)=$ $P_{(v)}\left(a \amalg P_{(v)}(b)\right)+P_{(v)}\left(P_{(v)}(a) \amalg b\right)$.

\subsection{Quasi-shuffle product}

We recall the construction of quasi-shuffle algebras [26]. Let $X$ be a locally finite set, that is, $X$ is the disjoint union of finite sets $X_{n}, n \geq 1$. The elements of $X_{n}$ are defined to have degree $n$. Elements in $X$ are called letters and noncommutative monomials in the letters are called words. Define $\bar{X}=X \cup\{0\}$. Suppose that there is an operation

$$
[\cdot, \cdot]: \bar{X} \times \bar{X} \rightarrow \bar{X}
$$

with the properties

S0. $[a, 0]=0$ for all $a \in \bar{X}$;

Springer 
S1. $[a, b]=[b, a]$ for all $a, b \in \bar{X}$;

S2. $[[a, b], c]=[a,[b, c]]$ for all $a, b, c \in \bar{X}$;

S3. either $[a, b]=0$ for all $a, b \in \bar{X}$, or $\operatorname{deg}([a, b])=\operatorname{deg}(a)+\operatorname{deg}(b)$ for all $a, b \in$ $\bar{X}$.

We define a Hoffman set to be a locally finite set $X$ with a pairing (4) that satisfies conditions S0-S3. Even though the original paper [26] only considered $\mathbf{k}$ to be a subfield of $\mathbb{C}$, much of the construction goes through for any commutative ring $\mathbf{k}$. So we will work in this generality whenever possible. Consider the k-module underlying the noncommutative polynomial algebra $\mathfrak{A}=\mathbf{k}\langle X\rangle$, that is, the free $\mathbf{k}$-algebra generated by $X$. The identity 1 of $\mathfrak{A}$ is called the empty word.

Definition 2.2. Let $\mathbf{k}$ be a commutative ring and let $X$ be a Hoffman set. The quasishuffle product $*$ on $\mathfrak{A}$ is defined recursively by

- $1 * w=w * 1=w$ for any word $w$;

- $\left(a w_{1}\right) *\left(b w_{2}\right)=a\left(w_{1} *\left(b w_{2}\right)\right)+b\left(\left(a w_{1}\right) * w_{2}\right)+[a, b]\left(w_{1} * w_{2}\right)$, for any words $w_{1}, w_{2}$ and letters $a, b$.

When $[\cdot, \cdot]$ is identically zero, $*$ is the usual shuffle product $\amalg$ defined recursively in Eq. (3).

Theorem 2.3 ((Hoffman)[26]). (1) (A, *) is a commutative graded $\mathbf{k}$-algebra.

(2) When $[\cdot, \cdot] \equiv 0,(\mathfrak{A}, *)$ is the shuffle product algebra $(T(V), \amalg)$, where $V$ is the vector space generated by $X$.

(3) Suppose further $\mathbf{k}$ is a subfield of $\mathbb{C}$. Together with the deconcatenation comultiplication

$$
\Delta: \mathfrak{A} \rightarrow \mathfrak{A} \otimes \mathfrak{A}, w \mapsto \sum_{u v=w} u \otimes v
$$

where $u v$ is the concatenation of words, and counit

$$
\epsilon: \mathfrak{A} \rightarrow \mathbf{k}, w \mapsto \delta_{w, 1},
$$

$(\mathfrak{A}, *)$ becomes a graded, connected bialgebra, in fact a Hopf algebra. When $[a, b]=0$ for all $a, b \in \bar{X}$, the Hopf algebra is the shuffle product Hopf algebra.

2.3. Mixable shuffle product

We next turn to the construction of mixable shuffle algebras and their properties [23]. The adjective mixable suggests that certain elements in the shuffles can be mixed or merged. We first give an explicit formula of the product before giving a recursive definition which, under proper restrictions, will be seen to be equivalent to Hoffman's quasi-shuffle product.

Intuitively, to form the shuffle product, one starts with two decks of cards and puts together all possible shuffles of the two decks. Suppose a shuffle of the two decks is 
taken and suppose a card from the first deck is followed immediately by a card from the second deck, we allow the option to merge the two cards and call the result a mixable shuffle. To get the mixable shuffle product of the two decks of cards, one puts together all possible mixable shuffles.

Given an $(m, n)$-shuffle $\sigma \in S(m, n)$, a pair of indices $(k, k+1), 1 \leq k<m+n$, is called an admissible pair for $\sigma$ if $\sigma(k) \leq m<\sigma(k+1)$. Denote $\mathcal{T}^{\sigma}$ for the set of admissible pairs for $\sigma$. For a subset $T$ of $\mathcal{T}^{\sigma}$, call the pair $(\sigma, T)$ a mixable $(m, n)$ shuffle. Let $|T|$ be the cardinality of $T$. By convention, $(\sigma, T)=\sigma$ if $T=\emptyset$. Denote

$$
\bar{S}(m, n)=\left\{(\sigma, T) \mid \sigma \in S(m, n), T \subset \mathcal{T}^{\sigma}\right\}
$$

for the set of mixable $(m, n)$-shuffles.

Let $A$ be a commutative k-algebra not necessarily having an identity. We will define another product, the mixable shuffle product, on the tensor space

$$
T(A):=\bigoplus_{k \geq 0} A^{\otimes k}
$$

and use it to construct the free commutative Rota-Baxter algebra on $A$. For $a=$ $a_{1} \otimes \ldots \otimes a_{m} \in A^{\otimes m}, b=b_{1} \otimes \ldots \otimes b_{n} \in A^{\otimes n}$ and $(\sigma, T) \in \bar{S}(m, n)$, the element

$$
\sigma(a \otimes b ; T)=u_{\sigma(1)} \hat{\otimes} u_{\sigma(2)} \hat{\otimes} \ldots \hat{\otimes} u_{\sigma(m+n)} \in A^{\otimes(m+n-|T|)},
$$

where for each pair $(k, k+1), 1 \leq k<m+n$,

$$
u_{\sigma(k)} \hat{\otimes} u_{\sigma(k+1)}= \begin{cases}u_{\sigma(k)} u_{\sigma(k+1)}, & (k, k+1) \in T \\ u_{\sigma(k)} \otimes u_{\sigma(k+1)}, & (k, k+1) \notin T,\end{cases}
$$

is called a mixable shuffle of the words $a$ and $b$.

Now fix $\lambda \in \mathbf{k}$. Define, for $a$ and $b$ as above, the mixable shuffle product

$$
a \diamond^{+} b:=a \diamond_{\lambda}^{+} b=\sum_{(\sigma, T) \in \bar{S}(m, n)} \lambda^{|T|} \sigma(a \otimes b ; T) \in \bigoplus_{k \leq m+n} A^{\otimes k} .
$$

As in the case of the shuffle product, the operation $\diamond^{+}$extends to a commutative and associative binary operation on

$$
T^{+}(A):=\bigoplus_{k \geq 1} A^{\otimes k}=A \oplus A^{\otimes 2} \oplus \ldots
$$

Making it a commutative algebra without identity. Note that this is so even when $A$ has an identity. This is similar to the case of tensor algebra. In any case, we take the unitarization

$$
\amalg_{\lambda}^{+}(A):=\amalg_{\mathbf{k}, \lambda}^{+}(A):=\mathbf{k} \oplus \bigoplus_{k \geq 1} A^{\otimes k}=\mathbf{k} \oplus A \oplus A^{\otimes 2} \oplus \ldots,
$$


and obtain a commutative algebra with the identity $\mathbf{1}$ being $\mathbf{1}_{\mathbf{k}} \in \mathbf{k}$ [23]. We call it the mixable shuffle algebra.

Suppose $A$ has an identity $\mathbf{1}_{A}$. Define

$$
\amalg_{\lambda}(A):=\amalg_{\mathbf{k}, \lambda}(A):=A \otimes \amalg_{\mathbf{k}, \lambda}^{+}(A)
$$

to be the tensor product algebra. More precisely, the product $\diamond=\diamond_{\lambda}$ on $\amalg_{\lambda}(A)$ is defined by

$$
\left(a_{0} \otimes a\right) \diamond_{\lambda}\left(b_{0} \otimes b\right):=\left(a_{0} b_{0}\right) \otimes\left(a \diamond_{\lambda}^{+} b\right), a_{0}, b_{0} \in A, a, b \in \amalg^{+}(A)
$$

and is called the augmented mixable shuffle product. Thus we have the algebra isomorphism (embedding of the second tensor factor)

$$
\alpha:\left(\amalg_{\lambda}^{+}(A), \diamond_{\lambda}^{+}\right) \rightarrow\left(\mathbf{1}_{A} \otimes \amalg_{\lambda}^{+}(A), \diamond_{\lambda}\right)
$$

The pair of products $\diamond_{\lambda}^{+}$and $\diamond_{\lambda}$ is a special case of the double products in Rota-Baxter algebras. See the remark after Theorem 2.4.

Define the k-linear endomorphism $P_{A}:=P_{A, \lambda}$ on $\amalg_{\lambda}(A)$ by assigning

$$
\begin{aligned}
& P_{A}\left(a_{0} \otimes a\right)=\mathbf{1}_{A} \otimes a_{0} \otimes a, a \in A^{\otimes n}, n \geq 1, \\
& P_{A}\left(a_{0} \otimes c\right)=\mathbf{1}_{A} \otimes c a_{0}, c \in A^{\otimes 0}=\mathbf{k}
\end{aligned}
$$

and extending by additivity. Let $j_{A}: A \rightarrow \amalg_{\lambda}(A)$ be the canonical inclusion map. Call $\left(\amalg_{\lambda}(A), P_{A}\right)$ the (mixable) shuffle Rota-Baxter k-algebra on $A$ of weight $\lambda$. The following theorem was proved in [23].

Theorem 2.4. The shuffle Rota-Baxter algebra $\left(\amalg_{\lambda}(A), P_{A}\right)$, together with the natural embedding $j_{A}$, is a free commutative Rota-Baxter k-algebra on A of weight $\lambda$. More precisely, for any Rota-Baxter k-algebra $(R, P)$ of weight $\lambda$ and algebra homomorphism $f: A \rightarrow R$, there is a Rota-Baxter $\mathbf{k}$-algebra homomorphism $\tilde{f}:\left(\amalg_{\lambda}(A), P_{A}\right) \rightarrow(R, P)$ such that $f=\tilde{f} \circ j_{A}$.

The reader might find it unusual to see two products $\diamond_{\lambda}^{+}$and $\diamond_{\lambda}$ defined on the same underlying module $\oplus_{k \geq 1} A^{k}$. This is in fact typical in Rota-Baxter algebras. Let $(R, P)$ be a Rota-Baxter algebra of weight $\lambda$. Then

$$
x \star_{P} y:=x P(y)+P(x) y+\lambda x y, \forall x, y \in R,
$$

defines an associative product on $R$, and, together with the linear operator $P: R \rightarrow R$, gives another Rota-Baxter algebra structure on $R$. This is called the double structure on $(R, P)$, denoted by $\left(R, \star_{P}, P\right)$ to emphasize the different product. Furthermore, $P:\left(R, \star_{P}, P\right) \rightarrow(R, P)$ is a Rota-Baxter algebra homomorphism: $P\left(x \star_{P} \quad y\right)=$ $P(x) P(y)$. This property is known to mathematicians since the 1960 s. The Lie algebra variation was independently discovered by physicists working in classical integrable systems [42]. In general, we use the product on $R$ to obtain the "double product" 
$\star_{P}$. In the construction of the free Rota-Baxter algebra reviewed above, we did the opposite. We constructed the product $\diamond_{\lambda}^{+}$first and use it to obtain $\diamond_{\lambda}$. But $\diamond_{\lambda}^{+}$is the double product of $\diamond_{\lambda}$, that is,

$$
x \diamond_{\lambda}^{+} y=x \diamond_{\lambda} P_{A}(y)+P_{A}(x) \diamond_{\lambda} y+\lambda x \diamond_{\lambda} y .
$$

This is not immediately clear just from the definitions of the products, but follows by considering Theorem 2.4, which necessarily implies the map $P_{A}$ to be a weight $\lambda$ Rota-Baxter operator. For then we have

$$
\begin{aligned}
\mathbf{1}_{A} \otimes\left(x \diamond_{\lambda}^{+} y\right) & =\left(\mathbf{1}_{A} \otimes x\right) \diamond_{\lambda}\left(\mathbf{1}_{A} \otimes y\right) \\
& =P_{A}(x) \diamond_{\lambda} P_{A}(y) \\
& =P_{A}\left(x \diamond_{\lambda} P_{A}(y)+P_{A}(x) \diamond_{\lambda} y+\lambda x \diamond_{\lambda} y\right) \\
& =\mathbf{1}_{A} \otimes\left(x \diamond_{\lambda} P_{A}(y)+P_{A}(x) \diamond_{\lambda} y+\lambda x \diamond_{\lambda} y\right) .
\end{aligned}
$$

As $P_{A}$ is injective, we obtain Eq. (12). We will return to this property in the proof of Theorem 2.5.

\subsection{The connection}

We now establish the connection between quasi-shuffle product and mixable shuffle product.

Let $\mathbf{k}$ be a commutative ring with identity. Let $X=\cup_{n \geq 1} X_{n}$ be a Hoffman set. Then the pairing $[\cdot, \cdot]$ in (4) extends by k-linearity to a binary operation on the free k-module $A=\mathbf{k}\{X\}$ on $X$, making $A$ into a commutative k-algebra without identity. Further $A$ is graded, with homogeneous components $A_{n}=\mathbf{k}\left\{X_{n}\right\}$, the free $\mathbf{k}$-module generated by $X_{n}$. Let $\tilde{A}=\mathbf{k} \oplus A$ be the unitary $\mathbf{k}$-algebra spanned by $A$. Then $\tilde{A}=\mathbf{k}\{\tilde{X}\}$ where $\tilde{X}=\left\{\mathbf{1}_{\mathbf{k}}\right\} \cup X$ with $\mathbf{1}_{\tilde{A}}:=\left(\mathbf{1}_{\mathbf{k}}, 0\right)$ the identity of $\tilde{A}$. Here and in the rest of the paper, we will use $\mathbf{1}_{A}$ (instead of $\mathbf{1}_{\tilde{A}}$ ) to denote this identity of $\tilde{A}$. We will call $A$ (resp. $\tilde{A}$ ) the algebra (resp. unitary algebra) spanned by $X$.

With the notations in Eq. (7) and (8), we have embeddings

$$
\begin{aligned}
& \beta: \amalg_{\lambda}^{+}(A) \rightarrow \amalg_{\lambda}^{+}(\tilde{A}) \rightarrow \amalg_{\lambda}(\tilde{A}), \\
& a \quad \mapsto \quad a \quad \mapsto \mathbf{1}_{A} \otimes a \text {. }
\end{aligned}
$$

of k-algebras. Here the first embedding is induced by the embedding $A \hookrightarrow \tilde{A}$ and the second embedding is the natural one, $\amalg_{\lambda}^{+}(\tilde{A}) \rightarrow \amalg_{\lambda}(\tilde{A}):=\tilde{A} \otimes \amalg_{\lambda}^{+}(\tilde{A})$.

Theorem 2.5. For a Hoffman set $X$, the quasi-shuffle algebra $\mathfrak{A}=\mathbf{k}\langle X\rangle$ is isomorphic to the algebra $\amalg_{\mathbf{1}_{\mathbf{k}}^{+}}^{+}(A)$ and thus to the subalgebra $\mathbf{1}_{A} \otimes \amalg_{\mathbf{1}_{\mathbf{k}}}^{+}(A)$ of the free commutative Rota-Baxter algebra $\amalg_{\mathbf{1}_{\mathbf{k}}}(\tilde{A})$ of weight $\mathbf{1}_{\mathbf{k}}$.

Proof: We define

$$
f: X \rightarrow X \subseteq A=A^{\otimes 1} \subset \amalg_{\mathbf{1}_{\mathbf{k}}}^{+}(A)
$$


to be the canonical embedding. We note that both $\mathfrak{A}$, with the concatenation product, and $\amalg_{\mathbf{1}_{\mathbf{k}}}^{+}(A)$, with the tensor product, are the free unitary non-commutative $\mathbf{k}$-algebra on $X$, that is, the tensor algebra on $A$. Thus $f$ extends uniquely to an isomorphism $\bar{f}: \mathfrak{A} \rightarrow \amalg_{\mathbf{1}_{\mathbf{k}}}^{+}(A)$ of vector spaces such that for any letters $a_{1}, \cdots, a_{n} \in X$, we have

$$
\bar{f}\left(a_{1} \cdots a_{n}\right)=a_{1} \otimes \cdots \otimes a_{n} \in A^{\otimes n} .
$$

To prove that $\bar{f}$ is also an isomorphism between $\mathfrak{A}$, with the quasi-shuffle product $*$, and $\amalg_{\mathbf{1}_{\mathbf{k}}}^{+}(A)$, with the mixable shuffle product $\diamond_{\mathbf{1}_{\mathrm{k}}}^{+}$, we just need to show that $\bar{f}$ preserves the products. We first note that the recursive relation of $*$ in Definition 2.2 can be inductively defined as follows. For any $m, n \geq 1$ and $a:=a_{1} \cdots a_{m}, b:=b_{1} \cdots b_{n}$ with $a_{i}, b_{j} \in X, 1 \leq i \leq m, 1 \leq j \leq n$, define $a * b$ by induction on the sum $m+n$. Then $m+n \geq 2$. When $m+n=2$, we have $a=a_{1}$ and $b=b_{1}$. Define

$$
a * b=a_{1} b_{1}+b_{1} a_{1}+\left[a_{1}, b_{1}\right] .
$$

Assume that $a * b$ has been defined for $m+n \geq k \geq 2$ and consider $a$ and $b$ with $m+n=k+1$. Then $m+n \geq 3$ and so at least one of $m$ and $n$ is greater than 1 . Then we define

$a * b=a_{1} b_{1} \cdots b_{n}+b_{1}\left(a_{1} *\left(b_{2} \cdots b_{n}\right)\right)+\left[a_{1}, b_{1}\right] b_{2} \cdots b_{n}$, when $m=1, n \geq 2$,

$a * b=a_{1}\left(\left(a_{2} \cdots a_{m}\right) * b_{1}\right)+b_{1} a_{1} \cdots a_{m}+\left[a_{1}, b_{1}\right] a_{2} \cdots a_{m}$, when $m \geq 2, n=1$,

$$
\begin{array}{r}
a * b=a_{1}\left(\left(a_{2} \cdots a_{m}\right) *\left(b_{1} \cdots b_{n}\right)\right)+b_{1}\left(\left(a_{1} \cdots a_{m}\right) *\left(b_{2} \cdots b_{n}\right)\right) \\
+\left[a_{1}, b_{1}\right]\left(\left(a_{2} \cdots a_{m}\right) *\left(b_{2} \cdots b_{n}\right)\right), \text { when } m, n \geq 2 .
\end{array}
$$

Here the products by $*$ on the right hand side of each equation are well-defined by the induction hypothesis. Then we define the multiplication by $\mathbf{1}_{\mathbf{k}}$ by claiming that $\mathbf{1}_{\mathbf{k}}$ is the identity.

We now prove the multiplicity

$$
\bar{f}(a * b)=\bar{f}(a) \diamond_{\mathbf{1}_{\mathbf{k}}}^{+} \bar{f}(b) .
$$

by a similar induction on $m+n$. When $m+n=2$, then $m=n=1$. Hence by Eq. (14), we have

$$
\bar{f}\left(a_{1} * b_{1}\right)=\bar{f}\left(a_{1} b_{1}+b_{1} a_{1}+\left[a_{1}, b_{1}\right]\right)=a_{1} \otimes b_{1}+b_{1} \otimes a_{1}+\left[a_{1}, b_{1}\right] .
$$

This is precisely $\bar{f}\left(a_{1}\right) \diamond^{+} \bar{f}\left(b_{1}\right)=a_{1} \diamond^{+} b_{1}$ by Eq. (6) since the first two terms are the shuffles of $a_{1}$ and $b_{1}$ and the third term comes from the only admissible pair $(1,2)$ for the $(1,1)$-shuffle id $\in S(1,1)$. 
Assume that Eq. (18) has been proved for $m+n \geq k \geq 2$ and consider $a$ and $b$ with $m+n=k+1$. Then either $m=1$ and $n \geq 2$, or $m \geq 2$ and $n=1$, or $m \geq 2$ and $n \geq 2$. We will check Eq. (18) when $m \geq 2$ and $n \geq 2$. The other cases are similar.

By Eq. (9), we have

$$
\left(\mathbf{1}_{A} \otimes \bar{f}(a)\right) \diamond_{\mathbf{1}_{\mathbf{k}}}\left(\mathbf{1}_{A} \otimes \bar{f}(b)\right)=\mathbf{1}_{A} \otimes\left(\bar{f}(a) \diamond_{\mathbf{1}_{\mathbf{k}}}^{+} \bar{f}(b)\right) .
$$

On the other hand, by Theorem $2.4, P_{A}$ is a Rota-Baxter operator. So we have

$$
\begin{aligned}
\left(\mathbf{1}_{A} \otimes\right. & \left.\bar{f}(a)) \diamond_{\mathbf{1}_{\mathbf{k}}}\left(\mathbf{1}_{A} \otimes \bar{f}(b)\right)=P_{A}(\bar{f}(a)) \diamond_{\mathbf{1}_{\mathbf{k}}} P_{A}(\bar{f}(b)) \quad \text { (by Eq. }(11)\right) \\
= & P_{A}\left(\bar{f}(a) \diamond_{\mathbf{1}_{\mathbf{k}}} P_{A}(\bar{f}(b))+P_{A}(\bar{f}(a)) \diamond_{\mathbf{1}_{\mathbf{k}}} \bar{f}(a)+\bar{f}(a) \diamond_{\mathbf{1}_{\mathbf{k}}} \bar{f}(b)\right) \\
& \text { (by Rota }- \text { Baxter relation Eq. }(1)) \\
= & \mathbf{1}_{A} \otimes\left(\left(a_{1} \otimes \cdots \otimes a_{m}\right) \diamond_{\mathbf{1}_{\mathbf{k}}}\left(\mathbf{1}_{A} \otimes b_{1} \otimes \cdots \otimes b_{n}\right)\right. \\
& +\left(\mathbf{1}_{A} \otimes a_{1} \otimes \cdots \otimes a_{m}\right) \diamond_{\mathbf{1}_{\mathbf{k}}}\left(b_{1} \otimes \cdots \otimes b_{n}\right)+\left(a_{1} \otimes \cdots \otimes a_{m}\right) \diamond_{\mathbf{1}_{\mathbf{k}}}\left(b_{1}\right. \\
& \left.\left.\otimes \cdots \otimes b_{n}\right)\right) \\
& \left(\text { by definitions of } \bar{f} \text { and } P_{A}\right) \\
= & \mathbf{1}_{A} \otimes\left(a_{1} \otimes\left(\left(a_{2} \otimes \cdots \otimes a_{m}\right) \diamond_{\mathbf{1}_{\mathbf{k}}}^{+}\left(b_{1} \otimes \cdots \otimes b_{n}\right)\right)\right. \\
& +b_{1} \otimes\left(\left(a_{1} \otimes \cdots \otimes a_{m}\right) \diamond_{\mathbf{1}_{\mathbf{k}}}^{+}\left(b_{2} \otimes \cdots \otimes b_{n}\right)\right) \\
& \left.+\left[a_{1}, b_{1}\right] \otimes\left(\left(a_{2} \otimes \cdots \otimes a_{m}\right) \diamond_{\mathbf{1}_{\mathbf{k}}}^{+}\left(b_{2} \otimes \cdots \otimes b_{n}\right)\right)\right) \quad(\text { by Eq. }(9)) \\
= & \mathbf{1}_{A} \otimes\left(a_{1} \otimes \bar{f}\left(\left(a_{2} \cdots a_{m}\right) *\left(b_{1} \cdots b_{n}\right)\right)+b_{1} \otimes \bar{f}\left(\left(a_{1} \cdots a_{m}\right) *\left(b_{2} \cdots b_{n}\right)\right)\right. \\
= & \mathbf{1}_{A} \otimes\left(\bar{f}\left(\left(a_{1} \cdots a_{m}\right) *\left(b_{1} \cdots b_{n}\right)\right)\right) \quad(\text { by Eq. }(17)) . \\
& \left.+\left[a_{1}, b_{1}\right] \otimes \bar{f}\left(\left(a_{2} \cdots a_{m}\right) *\left(b_{2} \cdots b_{n}\right)\right)\right) \quad(\text { by induction hypothesis }) \\
= & \mathbf{1}_{A} \otimes \bar{f}\left(a_{1}\left(\left(a_{2} \cdots a_{m}\right) *\left(b_{1} \cdots b_{n}\right)\right)+b_{1}\left(\left(a_{1} \cdots a_{m}\right) *\left(b_{2} \cdots b_{n}\right)\right)\right. \\
& \left.\left.+\left[a_{1}, b_{1}\right]\left(\left(a_{2} \cdots a_{m}\right) *\left(b_{2} \cdots b_{n}\right)\right)\right) \quad(\text { by definition of } \bar{f})\right) \\
& \\
& \cdots
\end{aligned}
$$

Since the map $a \mapsto \mathbf{1}_{A} \otimes a$ is injective, we have $\bar{f}(a * b)=\bar{f}(a) \diamond_{\mathbf{1}_{\mathbf{k}}}^{+} \bar{f}(b)$. This completes the induction. Thus when $\lambda=1$, we have $\bar{f}(a * b)=\bar{f}(a) \diamond_{\mathbf{1}_{\mathbf{k}}}^{+} \bar{f}(b)$ for all words $a$ and $b$ with $m, n \geq 1$, and hence for all $a$ and $b$ with $m, n \geq 0$ since when $m=0$ or $n=0$, we have $a=1$ or $b=1$ and the multiplications through $*$ and $\diamond_{\mathbf{1}_{\mathrm{k}}}^{+}$are both given by the identity. This proves the first isomorphism. The second one then follows from Eq. (13).

The theorem can also be proved by showing that $\diamond^{+}$satisfies the same recursion relations (14)-(17) of $*$. We record these relations for later applications. 
Lemma 2.6. For $a:=a_{1} \otimes \cdots \otimes a_{m}$ and $b:=b_{1} \otimes \cdots \otimes b_{n}$ in $\amalg_{\lambda}(\tilde{A})$, we have

(i) $a \diamond^{+} b=a_{1} \otimes b_{1}+b_{1} \otimes a_{1}+\lambda\left[a_{1}, b_{1}\right]$, when $m, n=1$,

(ii) $a \diamond^{+} b=a_{1} \otimes b_{1} \otimes \cdots \otimes b_{n}+b_{1} \otimes\left(a_{1} \diamond^{+}\left(b_{2} \otimes \cdots \otimes b_{n}\right)\right)$ $+\lambda\left[a_{1}, b_{1}\right] \otimes b_{2} \otimes \cdots \otimes b_{n}$, when $m=1, n \geq 2$,

(iii) $a \diamond^{+} b=a_{1} \otimes\left(\left(a_{2} \otimes \cdots \otimes a_{m}\right) \diamond^{+} b_{1}\right)+b_{1} \otimes a_{1} \otimes \cdots \otimes a_{m}$ $+\lambda\left[a_{1}, b_{1}\right] \otimes a_{2} \otimes \cdots \otimes a_{m}$, when $m \geq 2, n=1$,

(iv) $a \diamond^{+} b=a_{1} \otimes\left(\left(a_{2} \otimes \cdots \otimes a_{m}\right) \diamond^{+}\left(b_{1} \otimes \cdots \otimes b_{n}\right)\right)$ $+b_{1} \otimes\left(\left(a_{1} \otimes \cdots \otimes a_{m}\right) \diamond^{+}\left(b_{2} \otimes \cdots \otimes b_{n}\right)\right)$ $+\lambda\left[a_{1}, b_{1}\right] \otimes\left(\left(a_{2} \otimes \cdots \otimes a_{m}\right) \diamond^{+}\left(b_{2} \otimes \cdots \otimes b_{n}\right)\right)$, when $m, n \geq 2$.

Proof: We will only prove the fourth equation. Verifications of the others are simpler. Using Eq. (9) and the Rota-Baxter operator $P_{A}(x)=\mathbf{1}_{A} \otimes x$, we have

$$
\begin{aligned}
\mathbf{1}_{A} \otimes\left(a \diamond^{+} b\right) & =\left(\mathbf{1}_{A} \otimes a\right) \diamond\left(\mathbf{1}_{A} \otimes b\right) \\
= & P_{A}(a) \diamond P_{A}(b) \\
= & P_{A}\left(a \diamond P_{A}(b)+P_{A}(a) \diamond b+\lambda a \diamond b\right) \\
= & P_{A}\left(a \diamond\left(\mathbf{1}_{A} \otimes b\right)+\left(\mathbf{1}_{A} \otimes a\right) \diamond b+\lambda a \diamond b\right) \\
= & \mathbf{1}_{A} \otimes\left(a_{1} \otimes\left(\left(a_{2} \otimes \cdots a_{m}\right) \diamond^{+} b\right)+b_{1} \otimes\left(a \diamond^{+}\left(b_{2} \otimes \cdots \otimes b_{n}\right)\right)\right. \\
& \left.\quad+\lambda\left[a_{1}, b_{1}\right] \otimes\left(\left(a_{2} \otimes \cdots \otimes a_{m}\right) \diamond^{+}\left(b_{2} \otimes \cdots \otimes b_{n}\right)\right)\right) .
\end{aligned}
$$

By the injectivity of $P_{A}$, we have (iv).

We now prove the following consequence of Theorem 2.5 and Theorem 2.3.

Corollary 2.7. Under the same assumptions of Theorem 2.5 and the additional assumption that $\mathbf{k}$ is a subfield of $\mathbb{C}$, for any $\lambda \in \mathbf{k}$, the subalgebra $\amalg_{\lambda}^{+}(A)$ of $\amalg_{\lambda}^{+}(\tilde{A})$ and the subalgebra $\mathbf{1}_{A} \otimes \amalg_{\lambda}^{+}(A)$ of $\amalg_{\lambda}(\tilde{A})$ are Hopf algebras.

In the next section, we will extend this Hopf algebra to a larger Hopf algebra in $\amalg_{\lambda}(\tilde{A})$.

Proof: Because of the isomorphism (10), we only need to prove for one of the subalgebras for any given $\lambda$. When $\lambda=1$, the first part follows from Theorem 2.5 and Theorem 2.3. When $\lambda=0$, the first part is well-known (see [26], for example).

Now assume $\lambda \neq 1,0$. We will construct an algebra isomorphism

$$
g: \mathbf{1}_{A} \otimes \amalg_{\lambda}^{+}(A) \rightarrow \mathbf{1}_{A} \otimes \amalg_{1}^{+}(A) .
$$

Then the Hopf algebra structure on the later algebra gives a Hopf algebra structure on the former one. 
We first note that, if $P$ is a Rota-Baxter operator of weight $\mathbf{1}_{\mathbf{k}}$ on an algebra, then $Q:=\lambda P$ is a Rota-Baxter operator of weight $\lambda$ on the same algebra. This is clear since multiplying $\lambda^{2}$ to the weight $\mathbf{1}_{\mathbf{k}}$ Rota-Baxter equation of $P$ :

$$
P(x) P(y)=P(x P(y))+P(P(x) y)+P(x y),
$$

we obtain the weight $\lambda$ Rota-Baxter equation of $Q$ :

$$
Q(x) Q(y)=Q(x Q(y))+Q(Q(x) y)+\lambda Q(x y) .
$$

This applies in particular to the free commutative Rota-Baxter algebra $\amalg_{\mathbf{1}_{\mathbf{k}}}(\tilde{A})$ of weight $\mathbf{1}_{\mathbf{k}}$. Thus $\left(\amalg_{\mathbf{1}_{\mathbf{k}}}(\tilde{A}), Q\right)$, where $Q=\lambda P_{A, \mathbf{1}_{\mathbf{k}}}$, is a Rota-Baxter algebra of weight $\lambda$. Here, to avoid confusion, we have used $P_{A, \mathbf{1}_{\mathrm{k}}}$ to denote the Rota-Baxter operator $P_{A}$ of weight $\mathbf{1}_{\mathbf{k}}$ and will use $P_{A, \lambda}$ to denote the Rota-Baxter operator of weight $\lambda$ on $\amalg_{\lambda}(\tilde{A})$. By Theorem 2.4, $\left(\amalg_{\lambda}(\tilde{A}), P_{A, \lambda}\right)$ is the free Rota-Baxter algebra over $\tilde{A}$. So the natural algebra embedding $f: A \rightarrow \tilde{A} \rightarrow \amalg_{\mathbf{1}_{\mathbf{k}}}(\tilde{A})$ induces a homomorphism

$$
\tilde{f}:\left(\amalg_{\lambda}(\tilde{A}), P_{A, \lambda}\right) \rightarrow\left(\amalg_{\mathbf{1}_{\mathbf{k}}}(\tilde{A}), Q\right)
$$

of Rota-Baxter algebras of weight $\lambda$, such that $\tilde{f}\left(a_{0}\right)=a_{0}$ for $a_{0} \in \tilde{A}$. We will use the following lemma.

Lemma 2.8. For any $n \geq 0$ and $a_{0}, \cdots, a_{n} \in \tilde{A}$, we have

$$
\tilde{f}\left(a_{0} \otimes \cdots \otimes a_{n}\right)=\lambda^{n}\left(a_{0} \otimes \cdots \otimes a_{n}\right) .
$$

Proof: We prove the equation by induction on $n \geq 0$. When $n=0$, we have $\tilde{f}\left(a_{0}\right)=$ $f\left(a_{0}\right)=a_{0}$, so the equation is true. Assume that the equation has been proved for $n=$ $k \geq 0$ and consider $a_{0} \otimes \cdots \otimes a_{k+1} \in \tilde{A}^{\otimes(k+1)} \subseteq \amalg_{\lambda}(\tilde{A})$. Then using the properties of $\tilde{f}, P_{A, \lambda}$ and $\diamond_{\lambda}$, together with the induction hypothesis, we have

$$
\begin{aligned}
\tilde{f}\left(a_{0} \otimes \cdots \otimes a_{k+1}\right) & =\tilde{f}\left(a_{0} \diamond_{\lambda}\left(\mathbf{1}_{A} \otimes a_{1} \otimes \cdots \otimes a_{k+1}\right)\right) \\
& =\tilde{f}\left(a_{0} \diamond_{\lambda} P_{A, \lambda}\left(a_{1} \otimes \cdots \otimes a_{k+1}\right)\right) \\
& =\tilde{f}\left(a_{0}\right) \diamond_{\mathbf{1}_{\mathbf{k}}} Q\left(\tilde{f}\left(a_{1} \otimes \cdots \otimes a_{k+1}\right)\right) \\
& =a_{0} \diamond_{\mathbf{1}_{\mathbf{k}}} \lambda P_{A, \mathbf{1}_{\mathbf{k}}}\left(\lambda^{k} a_{1} \otimes \cdots \otimes a_{k+1}\right) \\
& =\lambda^{k+1} a_{0} \diamond_{\mathbf{1}_{\mathbf{k}}}\left(\mathbf{1}_{A} \otimes a_{1} \otimes \cdots \otimes a_{k+1}\right) \\
& =\lambda^{k+1} a_{0} \otimes \cdots \otimes a_{k+1} .
\end{aligned}
$$

This completes the induction.

Since $\lambda$ is invertible, $\tilde{f}$ has an inverse defined by

$$
f^{\prime}\left(a_{0} \otimes \cdots \otimes a_{n}\right)=\lambda^{-n} a_{0} \otimes \cdots \otimes a_{n}
$$


Therefore $\amalg_{\lambda}(\tilde{A})$ is isomorphic to $\amalg_{\mathbf{1}_{\mathbf{k}}}(\tilde{A})$. For $\mathbf{1}_{A} \otimes a_{1} \otimes \cdots \otimes a_{n} \in \mathbf{1}_{A} \otimes A^{\otimes n}$, we have

$$
\tilde{f}\left(\mathbf{1}_{A} \otimes a_{1} \otimes \cdots \otimes a_{n}\right)=\lambda^{n} \mathbf{1}_{A} \otimes a_{1} \otimes \cdots \otimes a_{n}=\mathbf{1}_{A} \otimes \lambda^{n} a_{1} \otimes \cdots \otimes a_{n}
$$

which is again in $\mathbf{1}_{A} \otimes A^{\otimes n}$. Similarly $f^{\prime}\left(\mathbf{1}_{A} \otimes A^{\otimes n}\right) \subseteq \mathbf{1}_{A} \otimes A^{\otimes n}$. Thus $\tilde{f}$ restricts to an isomorphism from $\mathbf{1}_{A} \otimes \amalg_{\lambda}^{+}(A)$ to $\mathbf{1}_{A} \otimes \amalg_{\mathbf{1}_{\mathbf{k}}^{+}}^{+}(A)$ and thus transfers the Hopf algebra structure from the image to the preimage.

\section{Hopf algebras in Rota-Baxter algebras}

We first recall the following theorem from [2].

Theorem 3.1 (Andrews-Guo-Keigher-Ono). For any commutative ring $\mathbf{k}$ with identity and for any $\lambda \in \mathbf{k}$, the free Rota-Baxter algebra $\amalg_{\lambda}(\mathbf{k})$ is a Hopf $\mathbf{k}$-algebra.

As shown in [2], when $\lambda=0$, we have the divided power Hopf algebra.

We now extend this result to $\amalg(\tilde{A})$ for a $\mathbf{k}$-algebra $\tilde{A}$ coming from a Hoffman set $X$. To avoid confusion, we will use $\mathbf{1}_{\mathbf{k}}$ for the identity of $\mathbf{k}$ and $\mathbf{1}_{A}$ for the identity of $\tilde{A}$ even though they are often identified under the structure map $\mathbf{k} \rightarrow \tilde{A}$ of the unitary k-algebra $\tilde{A}$.

Fix a $\lambda \in \mathbf{k}$. First note that, as a k-module,

$$
\amalg^{+}(\mathbf{k})=\bigoplus_{n \geq 0} \mathbf{k}^{\otimes n}=\mathbf{k} \oplus \mathbf{k} \oplus \mathbf{k}^{\otimes 2}+\cdots .
$$

There are two copies of $\mathbf{k}$ in the sum since $\mathbf{k}^{\otimes 0}=\mathbf{k} \cong \mathbf{k}^{\otimes 1}$. The identity of $\amalg^{+}(\mathbf{k})$ is the identity in the first copy, which we denote by $\mathbf{1}_{\mathbf{k}}^{\otimes 0}=\mathbf{1}$ as we did in (7). The second copy of $\mathbf{k}$, as well as its tensor powers $\mathbf{k}^{\otimes n}, n \geq 2$, are tensor powers of $\mathbf{k}$-modules. They are isomorphic as $\mathbf{k}$-modules, but not identical. If we take $\mathbf{1}_{\mathbf{k}}$ as a $\mathbf{k}$-basis of $\mathbf{k}$, then we have

$$
\amalg^{+}(\mathbf{k})=\mathbf{k} \mathbf{1} \oplus \mathbf{k} \mathbf{1}_{\mathbf{k}} \oplus \mathbf{k} \mathbf{1}_{\mathbf{k}}^{\otimes 2} \oplus \cdots=\bigoplus_{n \geq 0} \mathbf{k} \mathbf{1}_{\mathbf{k}}^{\otimes n},
$$

where $\mathbf{1}_{\mathbf{k}}^{\otimes k}, k \geq 1$, are tensor powers of the vector $\mathbf{1}_{\mathbf{k}}$. Then

$$
\amalg(\mathbf{k})=\mathbf{k} \otimes \amalg^{+}(\mathbf{k})=\mathbf{k}\left(\mathbf{1}_{\mathbf{k}} \otimes \mathbf{1}\right) \oplus \bigoplus_{n \geq 1} \mathbf{k}\left(\mathbf{1}_{\mathbf{k}} \otimes \mathbf{1}_{\mathbf{k}}^{\otimes n}\right)
$$

with the identity $\mathbf{1}_{\mathbf{k}} \otimes \mathbf{1}$. Since $\mathbf{k}$ is the base ring, the algebra homomorphism (10) gives

$$
\alpha:\left(\amalg^{+}(\mathbf{k}), \diamond^{+}\right) \cong\left(\mathbf{1}_{\mathbf{k}} \otimes \amalg^{+}(\mathbf{k}), \diamond\right) \cong(\amalg(\mathbf{k}), \diamond)
$$


Thus, by Theorem 3.1 we get

Lemma 3.2. For any $\lambda \in \mathbf{k},\left(\amalg^{+}(\mathbf{k}), \diamond^{+}\right)$is a Hopf algebra.

For now let $\tilde{A}$ be any unitary k-algebra with unit $\mathbf{1}_{A}$. Then

$$
\amalg^{+}(\tilde{A})=\bigoplus_{n \geq 0} \tilde{A}^{\otimes n}=\mathbf{k} \mathbf{1} \oplus \tilde{A} \oplus \tilde{A}^{\otimes 2} \oplus \cdots
$$

and

$$
\amalg(\tilde{A})=\tilde{A} \otimes \amalg^{+}(\tilde{A})=(\tilde{A} \otimes \mathbf{k} \mathbf{1}) \oplus \tilde{A}^{\otimes 2} \oplus \tilde{A}^{\otimes 3} \cdots .
$$

Since $\amalg(\tilde{A})$ is an $\tilde{A}$-algebra, and hence a k-algebra, we have the structure map $\gamma$ : $\mathbf{k} \rightarrow \amalg(\tilde{A})$ given by $\gamma(c)=c \mathbf{1}_{A} \otimes \mathbf{1}$. By the universal property of the free $\mathbf{k}$-RotaBaxter algebra $\amalg(\mathbf{k})$, we have an induced homomorphism $\gamma: \amalg(\mathbf{k}) \rightarrow \amalg(\tilde{A})$ of Rota-Baxter algebras. It is given by [23]

$$
\gamma\left(\mathbf{1}_{\mathbf{k}} \otimes \mathbf{1}_{\mathbf{k}}^{\otimes n}\right)=\mathbf{1}_{A} \otimes \mathbf{1}_{A}^{\otimes n}, n \geq 0
$$

Let

$$
\gamma^{+}: \amalg^{+}(\mathbf{k}) \rightarrow \amalg^{+}(\tilde{A}), \mathbf{1}_{\mathbf{k}}^{\otimes n} \mapsto \mathbf{1}_{A}^{\otimes n}, n \geq 0 .
$$

We have the following commutative diagram

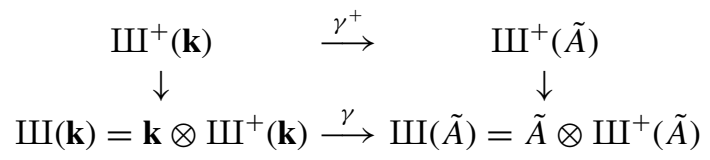

where the vertical arrow are the injective maps to the second tensor factors.

Theorem 3.3. Let $\mathbf{k} \subseteq \mathbb{C}$ be a field. Let $X$ be a Hoffman set and let the algebras $A$ and $\tilde{A}$ be the algebra and unitary algebra generated by $X$ (as defined before Theorem 2.5). Let $\lambda \in \mathbf{k}$.

(1) The algebra product of $\gamma^{+}\left(\amalg^{+}(\mathbf{k})\right)$ and $\amalg^{+}(A)$ in $\amalg^{+}(\tilde{A})$ has a Hopf algebra structure that expands the Hopf algebra structures on $\gamma^{+}\left(\mathrm{W}^{+}(\mathbf{k})\right)$ (see Lemma 3.2) and $\mathrm{W}^{+}(A)$ (see Corollary 2.7).

(2) The algebra product of $\gamma(\amalg(\mathbf{k}))$ and $\mathbf{1}_{A} \otimes \amalg^{+}(A)$ in $\amalg(\tilde{A})$ has a Hopf algebra structure that expands the Hopf algebra structures on $\gamma(\amalg(\mathbf{k}))$ (see Theorem 3.1) and $\mathbf{1}_{A} \otimes \amalg^{+}(A)$ (see Corollary 2.7).

See Theorem 3.6 for a characterization of the elements in these Hopf algebras. Springer 
Proof: Since the the isomorphism $\alpha: \amalg^{+}(\tilde{A}) \rightarrow \mathbf{1}_{A} \otimes \amalg^{+}(\tilde{A})$ in (10) restricts to isomorphisms $\gamma^{+}\left(\amalg^{+}(\mathbf{k})\right) \rightarrow \gamma(\amalg(\mathbf{k}))$ and $\amalg^{+}(A) \rightarrow \mathbf{1}_{A} \otimes \amalg^{+}(A)$, we only need to prove the first statement. Since the tensor product of two commutative, cocommutative Hopf algebras is a Hopf algebra [34, 32], assuming Proposition 3.4 which is stated and proved below, we see that $\gamma^{+}\left(\amalg^{+}(\mathbf{k})\right) \diamond^{+}\left(\mathbf{1} \otimes \amalg^{+}(A)\right)$ is a Hopf algebra for any $\lambda \in \mathbf{k}$ by Theorem 3.1 and Corollary 2.7 .

Proposition 3.4. For any weight $\lambda \in \mathbf{k}$, let $\diamond^{+}$be the mixable shuffle product of weight $\lambda$. The two subalgebras $\gamma^{+}\left(\amalg^{+}(\mathbf{k})\right)$ and $\amalg^{+}(A)$ of $\amalg^{+}(\tilde{A})$ are linearly disjoint. Therefore, $\gamma^{+}\left(\amalg^{+}(\mathbf{k})\right) \diamond^{+} \amalg^{+}(A)$ is isomorphic to the tensor product $\gamma(\amalg(\mathbf{k})) \otimes$ $\left(\mathbf{1}_{A} \otimes \amalg^{+}(A)\right)$.

Proof: Let $\mathbf{k} \subseteq \mathbb{C}, X, \tilde{X}, A$ and $\tilde{A}$ be as in Theorem 3.3. Since $X$ is locally finite, it is countable. So we can write $X=\left\{y_{n} \mid n \geq 1\right\}$. Also denote $y_{0}=\mathbf{1}_{A}$, the unit of $\tilde{A}$. Thus $\tilde{X}=\left\{y_{n} \mid n \in \mathbb{N}\right\}$ and $\tilde{A}=\oplus_{n \geq 0} \mathbf{k} y_{n}$. For $r \geq 1$ and $I=\left(i_{1}, \cdots, i_{r}\right) \in \mathbb{N}^{r}$, denote $y_{\otimes I}=y_{i_{1}} \otimes \cdots \otimes y_{i_{r}}$. Then

$$
A^{\otimes r}=\bigoplus_{I \in \mathbb{N}_{>0}^{r}} \mathbf{k} y_{\otimes I}, \quad \tilde{A}^{\otimes r}=\bigoplus_{I \in \mathbb{N}^{r}} \mathbf{k} y_{\otimes I} .
$$

By convention, we define $\mathbb{N}^{0}=\mathbb{N}_{>0}^{0}=\{\emptyset\}$, and $y_{\otimes \emptyset}=\mathbf{1}$. Let $\mathcal{I}=\cup_{r \geq 0} \mathbb{N}_{>0}^{r}$ and $\tilde{\mathcal{I}}=$ $\cup_{r \geq 0} \mathbb{N}^{r}$. We then have

$$
\amalg^{+}(A)=\bigoplus_{I \in \mathcal{I}} \mathbf{k} y_{\otimes I}, \quad \amalg^{+}(\tilde{A})=\bigoplus_{I \in \tilde{\mathcal{I}}} \mathbf{k} y_{\otimes I} .
$$

Recall that

$$
\gamma^{+}\left(\amalg^{+}(\mathbf{k})\right)=\bigoplus_{n \geq 0} \mathbf{k} \mathbf{1}_{A}^{\otimes n} .
$$

So to prove that $\oplus_{n \geq 0} \mathbf{k} \mathbf{1}_{A}^{\otimes n}$ and $\amalg^{+}(A)$ are linearly disjoint under the product $\diamond^{+}$, we only need to prove

Claim 3.1. The set $\left\{\mathbf{1}_{A}^{\otimes n} \diamond^{+} y_{\otimes I} \mid n \geq 0, I \in \mathcal{I}\right\}$ is linearly independent.

Before proceeding further, we give a formula for the product $\mathbf{1}_{A}^{\otimes n} \diamond^{+} y_{\otimes I}$ which expresses a mixable shuffle product as a sum of shuffle products in Eq. (2).

Lemma 3.5. For any $m \geq 0$ and $I \in \mathcal{I}$, we have

$$
\mathbf{1}_{A}^{\otimes m} \diamond^{+} y_{\otimes I}=\sum_{i=0}^{m} \lambda^{i}\left(\begin{array}{l}
n \\
i
\end{array}\right) \mathbf{1}_{A}^{\otimes(m-i)} \amalg y_{\otimes I} .
$$


Proof: Define the length of $I \in \mathbb{N}^{r}$ to be $\ell(I)=r$. We will prove by induction on $w=m+\ell(I)$. When $w=0$, we have $m=\ell(I)=0$. Then $\mathbf{1}_{A}^{\otimes m}$ and $y_{\otimes I}$ are both $\mathbf{1}$, so the lemma is clear, as it is if either $m=0$ or $\ell(I)=0$. Suppose it holds for all $\mathbf{1}_{A}^{\otimes m} \diamond^{+}$ $y_{\otimes I}$ with $m+\ell<w$. For given $\mathbf{1}_{A}^{\otimes m}$ and $y_{\otimes I}=y_{i_{1}} \otimes \cdots \otimes y_{i_{r}}$ with $m \geq 1, r \geq 1$ and $m+r=w$, let $y^{\prime}=y_{i_{2}} \otimes \cdots \otimes y_{i_{r}}$ if $r>1$ and $y^{\prime}=\mathbf{1}$ if $r=1$. Applying the recursive relation of $\diamond^{+}$in Eq. (19), the induction hypothesis, the Pascal equality and the recursive relation of $\amalg$ in Eq. (3), we have

$$
\begin{aligned}
& \mathbf{1}_{A}^{\otimes m} \diamond^{+} y_{\otimes I}=\mathbf{1}_{A} \otimes\left(\mathbf{1}_{A}^{\otimes(m-1)} \diamond^{+} y_{\otimes I}\right)+y_{i_{1}} \otimes\left(\mathbf{1}_{A}^{\otimes m} \diamond^{+} y^{\prime}\right)+\lambda y_{i_{1}} \otimes\left(\mathbf{1}_{A}^{\otimes(m-1)} \diamond^{+} y^{\prime}\right) \\
& =\mathbf{1}_{A} \otimes\left(\sum_{i=0}^{m-1} \lambda^{i}\left(\begin{array}{l}
n \\
i
\end{array}\right) \mathbf{1}_{A}^{\otimes(m-1-i)} \amalg y_{\otimes I}\right)+y_{i_{1}} \otimes\left(\sum_{i=0}^{m} \lambda^{i}\left(\begin{array}{c}
n-1 \\
i
\end{array}\right) \mathbf{1}_{A}^{\otimes(m-i)} \amalg y^{\prime}\right) \\
& +\lambda y_{i_{1}} \otimes\left(\sum_{i=0}^{m-1} \lambda^{i}\left(\begin{array}{c}
n-1 \\
i
\end{array}\right) \mathbf{1}_{A}^{\otimes(m-1-i)} \amalg y^{\prime}\right) \\
& \mathbf{1}_{A} \otimes\left(\sum_{i=0}^{m-1} \lambda^{i}\left(\begin{array}{l}
n \\
i
\end{array}\right) \mathbf{1}_{A}^{\otimes(m-1-i)} \amalg y_{\otimes I}\right)+y_{i_{1}} \otimes\left(\sum_{i=0}^{m} \lambda^{i}\left(\begin{array}{c}
n-1 \\
i
\end{array}\right) \mathbf{1}_{A}^{\otimes(m-i)} \amalg y^{\prime}\right) \\
& +y_{i_{1}} \otimes\left(\sum_{i=1}^{m} \lambda^{i}\left(\begin{array}{c}
n-1 \\
i-1
\end{array}\right) \mathbf{1}_{A}^{\otimes(m-i)} \amalg y^{\prime}\right) \\
& \mathbf{1}_{A} \otimes\left(\sum_{i=0}^{m-1} \lambda^{i}\left(\begin{array}{l}
n \\
i
\end{array}\right) \mathbf{1}_{A}^{\otimes(m-1-i)} \amalg y_{\otimes I}\right)+y_{i_{1}} \otimes\left(\sum_{i=0}^{m} \lambda^{i}\left(\begin{array}{l}
n \\
i
\end{array}\right) \mathbf{1}_{A}^{\otimes(m-i)} \amalg y^{\prime}\right) \\
& \stackrel{(3)}{=} \sum_{i=0}^{m-1} \lambda^{i}\left(\begin{array}{l}
n \\
i
\end{array}\right) \mathbf{1}_{A}^{\otimes(m-i)} \amalg y_{\otimes I}+\lambda^{m} y \otimes\left(\begin{array}{l}
n \\
m
\end{array}\right) \mathbf{1} \amalg y^{\prime} \text {. }
\end{aligned}
$$

Since $y_{i_{1}} \otimes\left(\mathbf{1} \amalg y^{\prime}\right)=y_{i_{1}} \otimes y^{\prime}=y=\mathbf{1}_{A}^{\otimes 0} \amalg y$, we get exactly what we want.

We continue with the proof of Proposition 3.4. For $r \geq 1$, let $[r]=(1, \cdots, r)$. For a sequence $I=\left(i_{1}, \cdots, i_{r}\right) \in \mathbb{N}^{r}$, denote $\operatorname{SSupp}(I)$ (called sequential support) for the subsequence (with ordering) of $I$ of non-zero entries. For an all zero sequence $I=(0, \cdots, 0)$ and the empty sequence $\emptyset$, we define $\operatorname{SSupp}(I)=\emptyset$. We then get a map

$$
\text { S Supp }: \tilde{\mathcal{I}} \rightarrow \tilde{\mathcal{I}}
$$

Clearly, $\mathcal{I}=\{I \in \tilde{\mathcal{I}} \mid \operatorname{SSupp}(I)=I\}$. So

$$
\tilde{\mathcal{I}}=\bigcup_{I \in \mathcal{I}} \operatorname{SS}_{\operatorname{Supp}}^{-1}(I)
$$


For each $I \in \mathcal{I}$, consider the subset $\mathcal{O}_{I}=\left\{y_{\otimes J} \mid J \in \operatorname{SSupp}^{-1}(I)\right\}$. Then we have

$$
\left\{y_{\otimes I} \mid I \in \tilde{\mathcal{I}}\right\}=\bigcup^{\bullet} \mathfrak{\bigcup}_{I \in \mathcal{I}} \mathcal{O}_{I}
$$

So $\mathcal{O}_{I}$ span linearly independent subspaces of $\amalg(\tilde{A})$.

By Lemma $3.5, \mathbf{1}_{A}^{\otimes n} \diamond^{+} y_{\otimes I}, n \geq 0$, is in the linear span of $\mathcal{O}_{I}$. Thus to prove Claim 3.1 and hence Proposition 3.4, we only need to prove that, for a fix $I \in \mathcal{I}$, the subset $\left\{\mathbf{1}_{A}^{\otimes n} \diamond^{+} y_{\otimes I} \mid n \geq 0\right\}$ is linearly independent.

Suppose the contrary. Then there are integers $n_{1}>n_{2}>\cdots>n_{r} \geq 0$ and $c_{1} \neq 0$ in $\mathbf{k}$ such that $\sum_{i=1}^{r} c_{i} \mathbf{1}_{A}^{\otimes n_{i}} \diamond^{+} y_{I}=0$. Express this sum as a linear combination in terms of the basis $\mathcal{O}_{I}$. By Lemma 3.5, the coefficient of $\mathbf{1}_{A}^{\otimes n_{1}} \otimes y_{I}$ is $c_{1}$, so we must have $c_{1}=0$, a contradiction.

It is desirable to characterize the elements in the Hopf algebra $\gamma^{+}\left(\amalg^{+}(\mathbf{k})\right) \diamond^{+}$ $\amalg^{+}(A)$. This is our last goal in this article. Recall that the length of $y_{\otimes I}$ with $I \in \mathbb{N}^{r}, r \geq 0$, is defined to be $\ell\left(y_{\otimes r}\right)=\ell(I)=r$. For a given $I \in \mathbb{N}^{n}$, the sum $\sum y_{\otimes J}$ over $J \in \mathbb{N}^{n}$ with $\operatorname{SSupp}(J)=\operatorname{SSupp}(I)$, is called the one-shuffled element of $y_{\otimes I}$, denoted by $O\left(y_{\otimes I}\right)$. So $O\left(y_{\otimes I}\right)$ is the sum over elements of $\mathcal{O}_{I}$ of length $\ell(I)$. For example, if $I=(2,0,1)$, then the corresponding one-shuffle element of $y_{\otimes I}=y_{2} \otimes \mathbf{1}_{A} \otimes y_{1}$ is $O\left(y_{\otimes I}\right)=y_{2} \otimes \mathbf{1}_{A} \otimes y_{1}+\mathbf{1}_{A} \otimes y_{2} \otimes y_{1}+y_{2} \otimes y_{1} \otimes \mathbf{1}_{A}$. On the other hand, $O\left(y_{\otimes I}\right)$ is $y_{\otimes I}$ if $I$ is either an all zero sequence or an all non-zero sequence. It is so named because the sum can be obtained from shuffling the subsequence of $y_{\otimes I}$ of the $\mathbf{1}_{A}$-entries with the subsequence of $I$ of the non- $\mathbf{1}_{A}$ entries (from SSupp $(I))$. To put it in another way, define a relation $\sim$ on $\tilde{\mathcal{I}}$ by $I_{1} \sim I_{2}$ if $\ell\left(I_{1}\right)=\ell\left(I_{2}\right)$ and $\operatorname{SSupp}\left(I_{1}\right)=\operatorname{SSupp}\left(I_{2}\right)$. Then it is easy to check that $\sim$ is an equivalence relation and a one-shuffled element is of the form $\sum y_{\otimes J}$ where the sum is taken over all $J$ in an equivalence class.

We now give another version of Theorem 3.3.

Theorem 3.6. Under the hypotheses of Theorem 3.3, the subspace of $\amalg^{+}(\tilde{A})$ spanned by one-shuffled elements form a Hopf algebra that contains the Hopf algebras $\gamma^{+}\left(\amalg^{+}(\mathbf{k})\right)$ and $\amalg^{+}(A)$.

By Theorem 3.3, we only need to prove the following lemma.

Lemma 3.7. The product of $\gamma^{+}\left(\amalg^{+}(\mathbf{k})\right)$ and $\amalg^{+}(A)$ in $\amalg^{+}(\tilde{A})$ is given by the subspace generated by one-shuffled elements.

Proof: To prove the lemma, let $U$ be the product of $\gamma^{+}\left(\amalg^{+}(\mathbf{k})\right)$ and $\amalg^{+}(A)$ in $\amalg^{+}(\tilde{A})$, and let $V$ be the subspace of one-shuffled elements of $\amalg^{+}(\tilde{A})$. Then by Lemma 3.5 and the comments before the theorem, we have $U \subseteq V$. To prove $V \subseteq U$, we only need to show that, for each $k \geq 0$ and $I \in\left(\mathbb{N}^{+}\right)^{n}, n \geq 0$, the one-shuffled element $\mathbf{1}_{A}^{\otimes k} \amalg x_{\otimes I}$ is in $U$. When $n=0, x_{\otimes I}=\mathbf{1}$. So $\mathbf{1}_{A}^{\otimes k} \amalg x_{\otimes I}=\mathbf{1}_{A}^{\otimes k}$ which is in $\gamma^{+}\left(\amalg^{+}(\mathbf{k})\right)$ and hence in $U$. When $n \geq 1$, we use induction on $k$. When $k=0$, then $\mathbf{1}_{A}^{\otimes k} \amalg x_{\otimes I}=x_{\otimes I}$ which is in $\amalg^{+}(A)$, hence is in $U$. Assume that it is true for 
$\mathbf{1}_{A}^{\otimes k}, k<m$ and consider $\mathbf{1}_{A}^{\otimes m} \amalg x_{\otimes I}$. By Lemma 3.5, we have

$$
\mathbf{1}_{A}^{\otimes m} \diamond^{+} y_{\otimes I}=\sum_{i=0}^{m} \lambda^{i}\left(\begin{array}{l}
n \\
i
\end{array}\right) \mathbf{1}_{A}^{\otimes(m-i)} \amalg y_{\otimes I} .
$$

The left hand side of the equation is in $U$ and, by induction, every term on the right hand side except the first one (with $i=0$ ) is also in $U$. Thus the first term, which is $\mathbf{1}_{A}^{\otimes m} \amalg y_{\otimes}$, is also in $U$. This completes the induction.

Acknowledgements We thank Zongzhu Lin and James Stasheff for helpful discussions and thank the referee for detailed comments that improved the clarity of the presentation. The first named author thanks the I.H.É.S. for warm hospitality, and the Ev. Studienwerk for financial support. The second named author acknowledges support from NSF grant DMS 0505643 and hospitality from the MPI for Mathematics at Bonn where the revision was completed.

\section{References}

1. M. Aguiar and J.-L. Loday, Quadri-algebras, J. Pure Applied Algebra, 191 (2004), $205-221$. arXiv:math.QA/03090171.

2. G. E. Andrews, L. Guo, W. Keigher and K. Ono, Baxter algebras and Hopf algebras, Trans. AMS, 355 (2003), no. 11, 4639-4656.

3. G. Baxter, An analytic problem whose solution follows from a simple algebraic identity, Pacific J. Math., 10 (1960), 731-742.

4. D. Bowman and D. M. Bradley, The algebra and combinatorics of shuffles and multiple zeta values, $J$. Combinatorial Theory Ser. A, 97 (1) (2002), 43-61. arXiv:math.CO/0310082

5. D. M. Bradley, Multiple $q$-zeta values, J. Algebra, 283 (2005), 752-798. arXiv:math.QA/0402093

6. P. Cartier, On the structure of free Baxter algebras, Adv. in Math., 9 (1972), 253-265.

7. K.T. Chen, Integration of paths, geometric invariants and a generalized Baker-Hausdorff formula, Ann. of Math., 65 (1957), 163-178.

8. A. Connes and D. Kreimer, Renormalization in quantum field theory and the Riemann-Hilbert problem. I. The Hopf algebra structure of graphs and the main theorem, Comm. Math. Phys., 210(1) (2000), 249273. arXiv:hep-th/9912092

9. A. Connes and D. Kreimer, Renormalization in quantum field theory and the Riemann-Hilbert problem. II. The $\beta$-function, diffeomorphisms and the renormalization group, Comm. Math. Phys., 216(1) (2001), 215-241. arXiv:hep-th/0003188

10. K. Ebrahimi-Fard, Loday-type algebras and the Rota-Baxter relation, Letters in Mathematical Physics, 61(2) (2002), 139-147.

11. K. Ebrahimi-Fard, On the associative Nijenhuis algebras, The Electronic Journal of Combinatorics, Volume 11(1), R38, (2004). arXiv:math-ph/0302062

12. K. Ebrahimi-Fard and L. Guo, On products and duality of binary, quadratic regular operads, J. Pure Applied Algebra, 200 (2005), 293-317. arXiv:math.RA/0407162

13. K. Ebrahimi-Fard and L. Guo, Rota-Baxter Algebras, Dendriform Algebras and Poincaré-BirkhoffWitt Theorem, preprint, arXiv:math.RA/0503342.

14. K. Ebrahimi-Fard and L. Guo, Rota-Baxter algebras and multiple zeta values, preprint, 2005, http://newark.rutgers.edu/ liguo.

15. K. Ebrahimi-Fard, L. Guo and D. Kreimer, Integrable Renormalization I: the ladder case, J. Math. Phys., 45 (2004), 3758-2769. arXiv:hep-th/0402095

16. K. Ebrahimi-Fard, L. Guo and D. Kreimer, Integrable Renormalization II: the general case, Annales Henri Poincaré, 6 (2005), 369-395. arXiv:hep-th/0403118

17. K. Ebrahim-Fard, L. Guo and D. Kreimer, Spitzer's Identity and the Algebraic Birkhoff Decomposition in pQFT, J. Phys. A: Math. Gen., 37 (2004), 11037-11052. arXiv:hep-th/0407082

18. F. Fares, Quelques constructions d'algèbres et de coalgèbres', Thesis, Université du Québec à Montréal.

19. A. B. Goncharov, Periods and mixed motives, preprint, arXiv:math.AG/0202154.

Springer 
20. L. Guo, Baxter algebra and differential algebra, in: Differential Algebra and Related Topics, World Scientific Publishing Company, (2002), 281-305. arXiv:math.RA/0407180

21. L. Guo, Baxter algebras and the umbral calculus, Adv. in Appl. Math., 27 (2-3) (2001), 405-426.

22. L. Guo, Baxter algebras, Stirling numbers and partitions, J. Algebra and Its Appl., 4 (2005), 153-164. arXiv:math.AC/0402348

23. L. Guo and W. Keigher, Free Baxter algebras and shuffle products, Adv. in Math., 150 (2000), 117-149. arXiv:math.RA/0407155

24. L. Guo and W. Keigher, On Baxter algebras: completions and the internal construction, Adv. in Math., 151 (2000), 101-127.

25. M. E. Hoffman, The algebra of multiple harmonic series, J. Algebra, 194(2), (1997), 477-495.

26. M. E. Hoffman, Quasi-shuffle products, J. Algebraic Combin., 11(1), (2000), 49-68.

27. M. E. Hoffman, Algebraic aspects of multiple zeta values, to appear in Zeta functions, topology and quantum physics, Springer-Verlag, 2005. arXiv: math.QA/0309425.

28. M. E. Hoffman, Y. Ohno, Relations of multiple zeta values and their algebraic expression, J. Algebra, 262 (2003), 332-347.

29. D. Kreimer, On the Hopf algebra structure of perturbative quantum field theories, Adv. Theor. Math. Phys., 2 (1998), 303-334. arXiv:q-alg/9707029

30. D. Kreimer, Chen's iterated integral represents the operator product expansion, Adv. Theor. Math. Phys., 3 (1999), 627-670. arXiv:hep-th/9901099

31. P. Leroux, Ennea-algebras, J. Algebra, 281 (2004), 287-302. arXiv:math.QA/0309213.

32. Z. Lin and D. Nakano, Representations of Hopf algebras arising from Lie algebras of Cartan type, $J$. Algebra, 189 (1997), 529-567.

33. J.-L. Loday and M. Ronco, Trialgebras and families of polytopes, in "Homotopy Theory: Relations with Algebraic Geometry, Group Cohomology, and Algebraic K-theory" Contemporary Mathematics 346 (2004), 369-398, arXiv:math.AT/0205043.

34. S. Montgomery, Hopf Algebras and Their Actions on Rings, American Mathematical Society, Providence, 1993.

35. C. Reutenauer, Free Lie Algebras, Oxford University Press, Oxford, 1993.

36. G.-C. Rota, Baxter algebras and combinatorial identities I, Bull. Amer. Math. Soc., 75 (1969), 325-329.

37. G.-C. Rota, Baxter algebras and combinatorial identities II, Bull. Amer. Math. Soc., 75 (1969), 330-334.

38. G.-C. Rota and D. A. Smith, Fluctuation theory and Baxter algebras, Symposia Mathematica, Vol. IX (Convegno di Calcolo delle Probabilitł, INDAM, Rome, 1971), pp. 179-201. Academic Press, London, (1972).

39. G.-C. Rota, Baxter operators, an introduction, In: Gian-Carlo Rota on Combinatorics, Introductory Papers and Commentaries, Joseph P.S. Kung, Editor, Birkhäuser, Boston, 1995.

40. G.-C. Rota, Ten mathematics problems I will never solve, Invited address at the joint meeting of the American Mathematical Society and the Mexican Mathematical Society, Oaxaca, Mexico, December 6, 1997. DMV Mittellungen Heft 2, 1998, 45-52.

41. F. Spitzer, A combinatorial lemma and its application to probability theory, Trans. Amer. Math. Soc., 82 (1956), 323-339.

42. M. A. Semenov-Tian-Shansky, Classical $r$-matrices, Lax equations, Poisson Lie groups and dressing transformations, In: Field theory, quantum gravity and strings, II (Meudon/Paris, 1985/1986), 174-214, Lecture Notes in Phys., 280 Springer, Berlin, (1987).

43. M. Sweedler, Hopf Algebras, Benjamin, New York, 1969. 